\title{
Scavenging ROS Decreases Amyloid-beta Levels via Activation of PI3K/Akt/GLUT1 pathway in N2a/APP695swe Cells
}

\section{Yan Peng}

Chongqing Medical University

\section{Li Zhang}

Chongqing Medical University

\section{Fanlin Zhou}

Chongqing University Cancer Hospital

\section{Yangyang Wang}

Chongqing University Cancer Hospital

\section{Shijie Li}

Chongqing University Cancer Hospital

\section{Jianing Fan}

Chongqing University Cancer Hospital

\section{Xiaoju Li}

Chongqing University Cancer Hospital

Yu Li ( $\triangle$ liyu100@cqu.edu.cn )

Chongqing Medical University https://orcid.org/0000-0003-1301-7234

\section{Research Article}

Keywords: Alzheimer's disease, Glucose metabolism, GLUT1, PI3K/Akt, ROS

Posted Date: December 10th, 2021

DOI: https://doi.org/10.21203/rs.3.rs-1148863/v1

License: (c) (1) This work is licensed under a Creative Commons Attribution 4.0 International License. Read Full License 


\section{Abstract}

Dysregulated glucose metabolism in the brain is considered to be the underlying cause of Alzheimer's disease (AD). Abnormal glucose metabolism in AD is associated with decreased glucose transporter 1 (GLUT1) and GLUT3 in the brain, but the underlying mechanisms remains unclear. Here, we reported that GLUT1 expression was decreased in N2a/APP695swe cells and GLUT3 expression was not significantly changed. Flow Cytometry analysis showed a significant increase of intracellular ROS content in N2a/APP695swe cells and GLUT1 expression was upregulated after treatment with the ROS scavenger Nacetyl-L-Cysteine (NAC). Cellular glucose uptake and ATP levels were reduced following decreased GLUT1 expression and increased after upregulating GLUT1. Western blot analyses showed that phosphorylation of PI3K/Akt pathway decreased in N2a/APP695swe cells. A 3 levels decreased after upregulation of GLUT1 expression and increased after downregulation of GLUT1. After NAC treatment, PI3K/Akt pathway phosphorylation levels and GLUT1 expression were upregulated, glucose uptake and ATP contents were increased, and A $\beta$ levels were decreased. After adding PI3K/Akt pathway inhibitor LY29004, GLUT1 expression was reduced and A $\beta$ levels were increased. Besides, the increased glucose uptake and ATP contents by the Akt activator SC79 were hindered with the GLUT1 inhibitor WZB117. A $\beta$ levels decreased after SC79 treatment and increased after WZB117 treatment. Overall, our data suggest that ROS reduced GLUT1 expression by inhibiting PI3K/Akt pathway activity resulting in impaired glucose metabolism and scavenging ROS prevents $A \beta$ via activation of PI3K/Akt/GLUT1 pathway in N2a/APP695swe cells.

\section{Introduction}

Alzheimer's disease (AD) is a progressive degenerative disease of the central nervous system and the main cause of dementia in the elderly ${ }^{[1]}$. The pathological characteristics of $A D$ mainly include $\beta$-amyloid $(A B)$ deposition, neurofibrillary tangles (NFTs), of which the neurotoxicity of $A \beta$ is currently recognized as the central part of $A D{ }^{[2]}$. Recent studies have found that the development of $A D$ is associated with metabolic abnormalities. Particularly, glucose metabolism disorders in the brain are considered to be responsible for the development of $A D{ }^{[3]}$. Impaired brain glucose uptake and utilization are closely associated with $A D$ pathological deterioration and cognitive impairment ${ }^{[4,5]}$.

Several studies have shown that the abnormal glucose metabolism in AD is mainly associated with a decrease in glucose transporter 1 (GLUT1) and GLUT3 in the brain, the main glucoses transporter responsible for glucose uptake in the mammalian brain ${ }^{[6]}$. The reduction of GLUT1 and GLUT3 might result in insufficient glucose uptake, compromised glucose metabolism, and ultimately lead to neuronal degeneration ${ }^{[3][7,6]}$. The Phosphoinositide 3-Kinase-Akt (PI3K/Akt) pathway has been suggested to play essential roles in the regulation of GLUT1 and GLUT3 ${ }^{[8]}$. Consistently, previous studies have found that abnormal PI3K/Akt signaling is involved in the development of $A D{ }^{[9,10]}$. However, little is currently known about the mechanisms underlying the abnormal PI3K/Akt signaling and reduced expression of GLUT1 and GLUT3 in AD. 
Reactive oxygen species (ROS) is an important factor in the early pathological development of AD [11]. ROS triggers the formation and accumulation of $A \beta$, meanwhile, $A \beta$ also promotes ROS production and enhances oxidative stress ${ }^{[12]}$. In addition, increased level of ROS is associated with decreased expression or activity of key proteins involved in the glucose metabolism pathway, which might result in a low energy state in neurons ${ }^{[13,14]}$. It has been reported that ROS affects brain energy metabolism by indirectly regulating neuronal cell permeability to glucose ${ }^{[1]}$. This study investigated the potential roles of ROS in the regulation of GLUT1 and GLUT3 and the underlying mechanisms in N2a/APP695swe cells.

\section{Materials And Methods}

\subsection{Cell culture and treatment}

The wild-type mouse neuroblastoma cells (N2a/WT) were purchased from Cell Resource Center, Shanghai Institutes for Biological Sciences, Chinese Academy of Sciences (Shanghai, China). N2a/APP695swe cells were purchased from Obio Technology (Shanghai, China). All cells were cultured in Dulbecco's modified Eagle medium (DMEM; Gibco, Waltham, MA, USA) containing 10\% fetal bovine serum (FBS, Gibco), and N2a/APP695swe cells medium were supplemented with an additional $0.04 \%$ puromycin $(5 \mathrm{mg} / \mathrm{ml})$ to screen cells stably expressing APP695swe. Cells were cultured at $5 \% \mathrm{CO}_{2}, 37^{\circ} \mathrm{C}$. These cells were treated with N-Acetyl-L-cysteine (NAC; 5 mM, Beyotime Institute of Biotechnology,

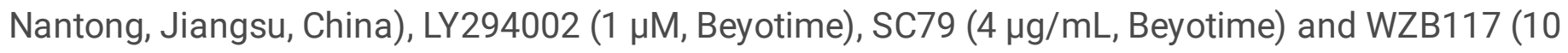
$\mu \mathrm{mol} / \mathrm{L}$; Selleck, Houston, Texas, USA), which were dissolved in dimethyl sulfoxide (DMSO; Solarbio, Beijing, China).

\subsection{Western blot}

Total cellular protein was extracted and quantified according to a standard procedure. Specifically, N2a/WT and N2a/APP695swe cells were homogenized in RIPA lysis buffer and protease inhibitors PMSF at $4^{\circ} \mathrm{C}$ for $30 \mathrm{~min}$, and centrifuged at $13,000 \mathrm{rpm}$ for $15 \mathrm{~min}$ at $4^{\circ} \mathrm{C}$ to collect the supernatants. The protein concentration was measured with the BCA assay reagent (Beyotime) according to the manufacturer's protocol. Following sodium dodecyl sulfate-polyacrylamide gel electrophoresis (SDSPAGE, Epizyme, Shanghai, China), the proteins were transferred onto a PVDF membrane (Solarbio). After saturation, the membranes were incubated at room temperature for 10 min in Quick Block ${ }^{\text {TM }}$ Western Sealing fluid (Beyotime) and subsequently incubated with primary antibodies against GLUT1 (1:100000, Abcam, Cambridge, MA, USA), GLUT3 (1:1000, Proteintech, Wuhan, China), Akt (1:1000), p-Akt (1:1000), PI3K (1:1000), p-PI3K (1:1000), or $\beta$-actin (1:1000), all from SAB (Singnalway Antibody, Maryland, USA), at $4^{\circ} \mathrm{C}$ overnight. The next day, peroxidase-conjugated IgG (Beyotime) was incubated for $40 \mathrm{~min}$ and enhanced chemiluminescence $(E C L)$ solution was subsequently applied to the membranes for detection of protein bands.

\subsection{Glucose uptake and measurement of ATP level in cells}


Glucose uptake by cells and the content of intracellular ATP were measured with Glucose Kit and ATP Assay Kit (Nanjing Jian Cheng, Nanjing, China). Briefly, after cells were treated as indicated for each experiment, cell samples preparation and detection procedures were done following the kit instruction.

\subsection{Cell viability CCK-8 assay}

Cell viability rates were measured using the CCK-8 Proliferation Assay Kit (Solarbio). Cells were seeded into 96-well plates at a density of 3000/well and grown overnight and then treated with $\operatorname{LY} 294002(5,10$, 20 or $40 \mu \mathrm{M}), \operatorname{SC79}(1,2,4$ or $8 \mu \mathrm{g} / \mathrm{mL})$, WZB117 $(1,5,10$ or $15 \mu \mathrm{mol} / \mathrm{L})$ or $\mathrm{NAC}(3,5,10$ or $15 \mathrm{mM})$ for up to $72 \mathrm{~h}$. At each time point, each group of cells was treated according to the instruction of the CCK-8 reagent.

\subsection{Flow cytometry ROS level assays}

According to the ROS Assay Kit (Beyotime) instruction, cells were loaded by an in-situ probe with DCFH$\mathrm{DA}$, and then cells were collected by centrifugation at $1000 \mathrm{rpm}$ for $5 \mathrm{~min}$. DCF fluorescence was detected by flow cytometry, which was the intracellular ROS level.

\subsection{Enzyme-linked immunosorbent assay (ELISA)}

The cell culture medium of each group was collected, and the content of $A \beta_{1-40}$ and $A \beta_{1-42}$ were detected according to the instruction of ELISA Kit (SAB, Maryland, USA).

\subsection{Statistical analysis}

All experiments were performed at least three times and statistical analysis was done using the SPSS13.0 package (SPSS Inc., Chicago, USA). The values for each group are expressed as the means \pm S.E.M. The ANOVA test was used whenever more than two groups were compared, and the significance level was set at $p<0.05$. A repeated measurement variance test was used to analyze time points comparisons. $P$ values of less than $0.05(p<0.05)$ were considered to be statistically significant.

\section{Results}

\subsection{Increased AB and ROS levels in N2a/APP695swe cells}

Compared with N2a/WT cells, $A \beta_{1-40}$ and $A \beta_{1-42}$ contents were increased in N2a/APP695swe cells (Fig. 1, $a+b$ ). Previous studies have reported that hypometabolism of glucose was most likely associated with reduced expression and function of key proteins in the glucose metabolic reaction pathway, which decreased with high levels of ROS ${ }^{[15,14]}$. Thus, we measured ROS levels by Flow Cytometry techniques, and the results showed that ROS concentration in N2a/APP695swe cells was strongly higher than that in N2a/WT cells (Fig. 1c).

Fig. 1 Increased A $\beta$ and ROS levels in N2a/APP695swe cells 
$(a+b)$ N2a/WT and N2a/APP695swe cells culture medium were collected to analyze A $\beta$ levels. The contents of $A \beta_{1-40}$ and $A \beta_{1-42}$ in the APP group were significantly higher than that in the WT group. (c) Intracellular ROS levels were detected by Flow Cytometry. As shown in the column, the ROS contents (P1

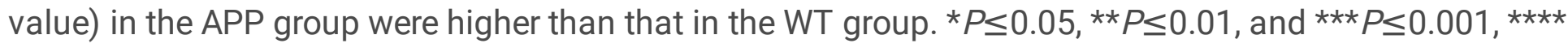
$P \leq 0.0001$.

\subsection{Decreased GLUT1 expression, the glucose uptake and ATP contents in N2a/APP695swe cells}

To detect whether the glucose metabolism of N2a/APP695swe cells was impaired, the glucose uptake and ATP contents of N2a/WT and N2a/APP695swe cells were detected respectively. Our results showed that glucose uptake and ATP contents of N2a/APP695swe cells were significantly lower than that of N2a/WT cells (Fig. 2, a + b). Considering that GLUT1 and GLUT3 are the main transporters responsible for the acquisition of glucose by nerve cells, we tested these two proteins to analyze the possible reasons for the impaired cellular glucose uptake. Western blots results indicated that compared with the WT group, the expression of GLUT1 in the APP group was decreased, and the difference was statistically significant (Fig. 2c), while the expression of GLUT3 was not changed, and the difference was not statistically significant (Fig. 2d).

Fig. 2 Decreased GLUT1 expression, the glucose uptake and ATP contents in N2a/APP695swe cells

The N2a/WT and N2a/APP695swe cells were collected separately from three different culture flasks and the proteins were extracted for the Western blot analysis. $(\mathbf{a}+\mathbf{b})$ Measurement of intracellular glucose uptake and ATP contents. $(\mathbf{c}+\mathbf{d})$ Western blot analysis of GLUT1 and GLUT3 proteins. GLUT1 expression was decreased in the APP group and GLUT3 was not significantly changed. Numbers 1,2 , and 3 represent proteins extracted at three different dates. ${ }^{*} P \leq 0.05$, $* * P \leq 0.01$, and ${ }^{*} * * \leq 0.001$.

\subsection{GLUT1 enhanced the glucose uptake and ATP contents, reduced AB levels in N2a/APP695swe cells}

To verify that the disordered cellular glucose metabolism was caused by low GLUT1 expression, we transfected N2a/WT and N2a/APP695swe cells with GLUT1-overexpressed plasmids, and western blot results showed the protein expression of GLUT1 after transfection (Fig. 3a). As we expected that after upregulated GLUT1, the glucose uptake and ATP contents were increased in both the WT and APP groups, with statistically significant differences (Fig. $3, b+c)$. Then we proceeded to test the $A \beta$ levels and the ELASA results showed that both $A \beta_{1-40}$ and $A \beta_{1-42}$ contents were reduced (Fig. $3, d+e$ ).

Fig. 3 GLUT1 enhanced the glucose uptake and ATP contents, reduced A 3 levels in N2a/APP695swe cells

The results from one of three experiments are shown. ${ }^{\star} P \leq 0.05,{ }^{\star} * P \leq 0.01$, and ${ }^{\star} * \star P \leq 0.001$. (a) GLUT1 overexpression plasmid was transfected into N2a/WT and N2a/APP695swe cells for $24 \mathrm{~h}$ and the GLUT1 protein level was detected by Western blot. $(\mathbf{b}+\mathbf{c})$ Measurement of intracellular glucose uptake and ATP level. After upregulated GLUT1, the glucose uptake and ATP contents were increased in both two groups. 
$(\mathbf{d}+\mathbf{e}) \mathrm{N} 2 \mathrm{a} / \mathrm{WT}$ and N2a/APP695swe cells culture medium were collected to analyze A $\beta$ levels. After overexpression of GLUT1, the contents of $A \beta_{1-40}$ and $A \beta_{1-42}$ were markedly reduced in the APP group.

\subsection{ROS scavenger N-acetyl-L-Cysteine (NAC) increased GLUT1 expression, the glucose uptake and ATP contents, decreased A $\beta$ levels in N2a/APP695swe cells}

Next, we treated the cells with the N-acetyl-L-Cysteine (NAC), which was commonly used as a scavenger of ROS. We explored four concentration gradients $(3,5,10,15 \mathrm{mM})$ according to the drug instruction of NAC and relevant literature ${ }^{[16]}$. The CCK-8 results showed that cells incubated with $5 \mathrm{mM}$ were the most active, and almost all cells died after incubation with NAC concentration greater than that (Fig. 4a). After NAC treatment for 24 h, ROS concentrations decreased in N2a/APP695swe cells (Fig. 4b). Western blot results showed that GLUT1 expression was significantly upregulated, compared with the control group (Fig. 4c), the glucose uptake and ATP contents also increased (Fig. 4, d +e). Meanwhile, the contents of

$A \beta_{1-40}$ and $A \beta_{1-42}$ in N2a/APP695swe cells decreased, with statistically significant differences (Fig. 4, $f+$ g).

Fig. 4 ROS scavenger N-acetyl-L-Cysteine (NAC) increased GLUT1 expression, the glucose uptake and ATP contents, decreased A $\beta$ levels in N2a/APP695swe cells

The results from one of three experiments are shown. ${ }^{*} P \leq 0.05$, $* * P \leq 0.01$, and ${ }^{*} * P \leq 0.001$. (a) The effect of NAC on viability in N2a/APP695swe cells was analyzed by the CCK-8 assay, $5 \mathrm{mM}$ was chosen as the correct concentration. (b) After NAC treatment for $24 \mathrm{~h}$, ROS concentrations decreased in N2a/APP695swe cells. (c) GLUT1 expression was analyzed by Western blot after being treated with NAC for $24 \mathrm{~h}$. $(\mathbf{d}+\mathbf{e})$ Measurement of intracellular glucose uptake and ATP levels. After NAC treatment for 24 $h$, the glucose uptake and ATP contents were increased. $(\mathbf{f}+\mathbf{g})$ After NAC treatment for $24 \mathrm{~h}$, the contents of $A \beta_{1-40}$ and $A \beta_{1-42}$ decreased in N2a/APP695swe cells.

\subsection{Phosphorylation of PI3K/Akt pathway decreased in N2a/APP695swe cells}

To clarify the mechanism by which ROS regulated GLUT1, we further tested the PI3K/Akt signal pathway, an important upstream pathway that controlled GLUT1. Our results suggested that the phosphorylation levels of PI3K and Akt in the APP group were lower than that in the WT group, and the difference was statistically significant. The total protein expressions of PI3K and Akt were not a significant variety (Fig. 5, $a+b)$.

Fig. 5 Phosphorylation of PI3K/Akt pathway decreased in N2a/APP695swe cells

The results from one of three experiments are shown. ${ }^{*} P \leq 0.05$, ${ }^{\star *} P \leq 0.01$, and ${ }^{*} * * \leq 0.001$. (a $\left.+\mathbf{b}\right)$ The level of the PI3K/Akt pathway was analyzed by Western blot. Numbers 1, 2, and 3 represent proteins extracted at three different dates. 


\subsection{NAC upregulated PI3K/Akt/GLUT1 pathway levels, increased the glucose uptake and ATP contents, decreased A $\beta$ levels}

Then we treated N2a/APP695swe cells with NAC and/or PI3K inhibitor LY294002. LY294002 is a potent inhibitor of PI 3-kinase activity based on the quercetin structure, which inhibits PI3K by reversibly binding to the ATP site of PI3K ${ }^{[17]}$. According to the drug instructions and literature ${ }^{[18]}$, we selected four concentration gradients $(5,10,20,40 \mu \mathrm{M})$ to screen the optimal concentration of LY294002 for N2a/APP695swe cells. The CCK-8 results showed that the cell viability was significantly reduced at 20 $\mu \mathrm{M}$ LY294002 concentration (Fig. 6a), whereas cells became shrunken when the concentration was 40 $\mu \mathrm{M}$ after $24 \mathrm{~h}$ treatment, thus we chose $20 \mu \mathrm{M}$ as the drug concentration. After NAC treatment we found that p-PI3K, p-Akt, and GLUT1 expression were increased, while decreased after LY294002 addition (Fig. 6b). Similarly, the glucose uptake and ATP contents were reduced after the cells were co-incubated with LY294002 in the medium containing NAC, with statistically significant differences (Fig. 6, c + d). Interestingly, the contents of $A \beta_{1-40}$ and $A \beta_{1-42}$ increased after the addition of LY294002 (Fig. 6, e + f). These data indicated that maintaining the activity of the PI3K/Akt/GLUT1 pathway is essential for A $\beta$ clearance.

Fig. 6 NAC upregulated PI3K/Akt/GLUT1 pathway levels, increased glucose uptake and ATP contents, decreased $A \beta$ levels

(a) The effect of LY294002 on viability in N2a/APP695swe cells was analyzed by the CCK-8 assay, $20 \mu \mathrm{M}$ LY294002 was chosen as the correct concentration. (b) The Western blot results for p-PI3K/Total-PI3K, $p$ Akt/Total-Akt, and GLUT1 after being treated with NAC and/or LY294002 for $24 \mathrm{~h}$. (c + d) The glucose uptake and ATP levels were increased after NAC treatment for $24 \mathrm{~h}$, while decreased after LY294002 addition. $(\mathbf{e}+\mathbf{f}) \mathrm{N} 2 \mathrm{a} / \mathrm{APP} 695$ swe cells culture medium was collected to analyze $A \beta$ levels. The contents of $A \beta_{1-40}$ and $A \beta_{1-42}$ were reduced after NAC treatment for $24 \mathrm{~h}$, while increased after $L Y 294002$ addition.

\subsection{Activated PI3K/Akt/GLUT1 increased the glucose uptake and ATP contents, decreased A $\beta$ levels in N2a/APP695swe cells}

To further demonstrate that the PI3K/Akt pathway was dependent on GLUT1 to improve glucose metabolism levels, we co-treated cells with SC79, an activator of Akt, and WZB117, a GLUT1-specific inhibitor. WZB117 binds to amino acid residues in the central channel region of GLUT1 to form three hydrogen bonds thereby inhibiting GLUT1 ${ }^{[19]}$. Likewise, we selected four concentration gradients $(1,2,4$, $8 \mu \mathrm{g} / \mathrm{mL}$ ) based on drug specification and literature ${ }^{[20]}$ to screen the optimal concentration of SC79 for cells. The CCK-8 results showed that the cell viability was increased at $4 \mu \mathrm{g} / \mathrm{mL} \mathrm{SC79}$ concentration (Fig. 7a). We also determined the best concentration of WZB117 was $10 \mu \mathrm{mol} / \mathrm{L}$ based on the instruction and CCK-8 assay results because most of the cells died when the WZB117 concentration was $15 \mu \mathrm{mol} / \mathrm{L}$ 
(Fig. 7b). After SC79 and WZB117 incubated cells together for $24 \mathrm{~h}$, glucose uptake and ATP contents were markedly decreased, and the differences were statistically significant (Fig. 7, $c+d)$. The contents of $A \beta_{1-40}$ and $A \beta_{1-42}$ were reduced after SC79 treatment for $24 \mathrm{~h}$, while increased after WZB117 addition (Fig. 7, e +f).

Fig. 7 Activated PI3K/Akt/GLUT1 increased glucose uptake and ATP contents, decreased A $\beta$ levels in N2a/APP695swe cells

The results from one of three experiments are shown. ${ }^{*} P \leq 0.05, * \star P \leq 0.01$, and $* \star \star P \leq 0.001$. (a) The effect of SC79 on viability in N2a/APP695swe cells was analyzed by the CCK-8 assay, $4 \mu \mathrm{g} / \mathrm{mL}$ SC79 was chosen as the correct concentration. (b) The effect of WZB117 on viability in N2a/APP695swe cells was analyzed by the CCK-8 assay, $10 \mu \mathrm{mol} / \mathrm{L}$ was chosen as the correct concentration. $(\mathbf{c}+\mathbf{d})$ The glucose uptake and ATP levels were increased after SC79 treatment for $24 \mathrm{~h}$, while decreased after WZB117 addition. $(\mathbf{e}+\mathbf{f}) \mathrm{N} 2 \mathrm{a} / \mathrm{APP} 695$ swe cells culture medium was collected to analyze $A \beta$ levels. The contents of $A \beta_{1-40}$ and $A \beta_{1-42}$ were reduced after SC79 treatment for $24 \mathrm{~h}$, while increased after WZB117 addition.

Fig. 8 Schematic: ROS reduces PI3K/Akt pathway phosphorylation levels leading to cellular GLUT1 deficiency and impaired glucose uptake, NAC improves this state

\section{Discussion}

The pathogenesis of $A D$ is complex, and many mechanisms are involved in the pathogenesis of $A D$ in a synergistic manner, such as $A \beta$ toxicity, oxidative stress, and impaired glucose metabolism ${ }^{\text {[21] [22] }}{ }^{\text {. During }}$ the stage of $A D$ development, the ability of the brain to utilize glucose is progressively impaired, even to the point of decreasing ATP production by $50 \%{ }^{[7]}$. Glucose is the main source of energy for the nervous system and only relies on specific transporter to cross the phospholipid bilayer into cells ${ }^{[23]}$. Glucose uptake in the brain is mainly reliant on GLUT1 and GLUT3, and is closely related to their levels $[24,25][26]$. Studies have reported that the accumulation of ROS, the reduction of GLUT1/GLUT3, and the impaired glucose metabolism all occurred in brain regions associated with memory and cognitive function ${ }^{[1]}{ }^{[5]}$. A previous study found that 4-Hydroxynonenal (HNE, an aldehyde product of membrane lipid peroxidation) impaired the glucose transport process, however, GLUT3 protein expression was not altered ${ }^{[27]}$. In the present study, we found that high levels of ROS and AB, GLUT1 expression was significantly reduced, while GLUT3 showed no significant change. We further showed by NAC treatment and overexpression experiments that in vitro ROS blocked glucose metabolism by decreasing GLUT1 expression. Therefore, it possible that GLUT1 is vulnerable to ROS attack during the shuttling and transport of glucose across the cytoplasm and cytosol, and eventually glucose uptake and glucose metabolism were impaired.

To investigate the mechanism of ROS-induced GLUT1 deficiency, we examined PI3K/Akt pathway levels in N2a/APP695swe cells. Our data showed that ROS reduced GLUT1 expression by suppressing PI3K/Akt pathway activity resulting in impaired glucose metabolism. The latest review summarized the role of $\mathrm{PI} 3 \mathrm{~K} /$ Akt pathway in $\mathrm{AD}$ and supported our results ${ }^{[28]}$. The article pointed out that abnormalities of the 
PI3K/Akt pathway, especially a decrease in Akt phosphorylation, prevented GLUT from transporting glucose into the cell and reduced ATP. Additional studies have also supported our results. Tahir Ali et al. investigated the antioxidant effects of anthocyanins in vivo and in vitro, and found that the reduction in $\mathrm{p}-\mathrm{PI} 3 \mathrm{~K} / \mathrm{Akt} / \mathrm{GSK} 3 \beta$ pathway levels was caused by ROS induced by amyloid $\beta$ oligomers $(\mathrm{A} \beta O)^{[10]}$. ROS could affect energy metabolism via the PI3K/Akt pathway, particularly Akt, which is a metabolically critical sensor ${ }^{[29]}$. Therefore, we believed that maintaining PI3K/Akt signaling pathway levels was helpful in protecting neuronal cells from damage by $A D$ risk factors.

$A \beta$ is produced by sequential cleavage of amyloid precursor protein (APP) by $\beta$-secretase and $\gamma$-secretase ${ }^{[30]}$. ROS could activate $\gamma$-secretase to trigger $A \beta$ production ${ }^{[31]}$. Under normal conditions, the production, degradation and clearance of $A \beta$ are in a dynamic equilibrium. However, various abnormalities lead to impaired $A \beta$ degradation or clearance, and total $A \beta$ production increased, while $A \beta$ generation did not

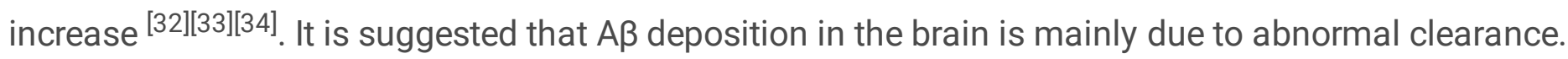
Oxidative stress could produce interference in the clearance of $A \beta$ by oxidizing the low-density lipoprotein receptor-related protein 1 (LRP1), a key $A \beta$ clearance transporter in the brain ${ }^{[35]}$. Interestingly, Winkler, E.A. et al. found that GLUT1 deficiency decreased $A \beta$ clearance by reducing LRP1 expression in vivo, thereby accelerating $A \beta$ deposition ${ }^{[36]}$. Here, we found that $A \beta$ levels were negatively correlated with PI3K/Akt/GLUT1 pathway levels and ATP. Thus, in the present study, scavenging ROS to reduce A $\beta$ was likely due to a certain degree of restored cellular energy supply and clearance of $A \beta$ being enhanced. Meanwhile, clearance of $A \beta$ required the PI3K/Akt/GLUT1 pathway to maintain ATP levels and that A $\beta$ accumulation correlated with GLUT1 levels. The specific molecular mechanisms require further investigation. Our data suggested that GLUT1 could work as a therapeutic target for AD, maintaining neuronal glucose metabolism and promoting the clearance of $A \beta$.

In conclusion, the present study found that ROS reduced GLUT1 expression by inhibiting PI3K/Akt pathway activity, which results in impaired glucose metabolism in N2a/APP695swe cells. Our results suggest that the PI3K/Akt/GLUT1 pathway might play a bridge role between $A \beta$, ROS, and neurometabolic disorders in AD. In future studies, we will continue to explore the function of GLUT1 (including GLUT3) in transporting glucose and the mechanisms regulating its movement between plasma membranes.

\section{Abbreviations}

$A D$, Alzheimer's disease

$A \beta$, Amyloid-beta

ATP, Adenosine triphosphate

GLUT, Glucose transporter

NAC, N-acetyl-L-Cysteine 
NFTs, Neurofibrillary tangles

N2a, Neuro-2a

WT, Wild type

p-Akt, Phosphorylated Akt

PI3K, Phosphoinositide 3-kinase

p-PI3K, Phosphorylated PI3K

ROS, Reactive oxygen species

\section{Declarations}

\section{Statements \& Declarations}

Ethics Approval and Consent to Participate: Not applicable.

Consent for Publication: All the authors agreed to publish this review.

Conflict of Interest: The authors declare no competing interests.

\section{Funding}

This work was supported by the National Natural Science Foundation of China (NSFC: 81671261) and the Natural Science Foundation of Chongqing (No. cstc2017jcyjAX0050).

\section{Acknowledgments}

We thank the technical support from the life science academy of Chongqing Medical University and thank the Center for Neuroscience Research School of Basic Medicine of Chongqing Medical University and the Department of Pathology of Chongqing University Cancer Hospital for help.

\section{Author contributions}

Yan Peng: Design, Conceptualization, Data curation, Formal analysis, Investigation, Methodology, Project administration, Resources, Software, Supervision, Writing-original draft, Writing-review \& editing.

Li Zhang: Design, Conceptualization, Data curation, Project administration, Resources, Supervision, Writing-review, and editing.

Fanlin Zhou: Design, Conceptualization, Data curation, Project administration, Writing-review, and editing. Yangyang Wang: Conceptualization, Data curation, Drawing, revision. 
Shijie Li, Jianing Fan, and Xiaoju Li: Conceptualization, revision.

Yu Li: Design, Conceptualization, revision.

\section{Data Availability}

All data generated or analysed during the current study are included in this published article.

\section{References}

1. Calabro M, Rinaldi C, Santoro G, Crisafulli C (2021) The biological pathways of Alzheimer disease: a review. AIMS Neurosci 8 (1):86-132. doi:10.3934/Neuroscience.2021005

2. Wang J, Gu BJ, Masters CL, Wang YJ (2017) A systemic view of Alzheimer disease - insights from amyloid-beta metabolism beyond the brain. Nat Rev Neurol 13 (10):612-623

3. Pujol-Gimenez J, Martisova E, Perez-Mediavilla A, Lostao MP, Ramirez MJ (2014) Expression of the glucose transporter GLUT12 in Alzheimer's disease patients. J Alzheimers Dis 42 (1):97-101. doi:10.3233/JAD-132498

4. Mosconi L, De Santi S, Li J, Tsui WH, Li Y, Boppana M, Laska E, Rusinek H, de Leon MJ (2008) Hippocampal hypometabolism predicts cognitive decline from normal aging. Neurobiol Aging 29 (5):676692. doi:10.1016/j.neurobiolaging.2006.12.008

5. Chen Z, Zhong C (2013) Decoding Alzheimer's disease from perturbed cerebral glucose metabolism: implications for diagnostic and therapeutic strategies. Prog Neurobiol 108:21-43.

doi:10.1016/j.pneurobio.2013.06.004

6. McEwen BS, Reagan LP (2004) Glucose transporter expression in the central nervous system: relationship to synaptic function. Eur J Pharmacol 490 (1-3):13-24. doi:10.1016/j.ejphar.2004.02.041

7. Szablewski L (2017) Glucose Transporters in Brain: In Health and in Alzheimer's Disease. J Alzheimers Dis 55 (4):1307-1320. doi:10.3233/JAD-160841

8. Li H, Lan J, Wang G, Guo K, Han C, Li X, Hu J, Cao Z, Luo X (2020) KDM4B facilitates colorectal cancer growth and glucose metabolism by stimulating TRAF6-mediated AKT activation. J Exp Clin Cancer Res 39 (1):12. doi:10.1186/s13046-020-1522-3

9. Perluigi M, Pupo G, Tramutola A, Cini C, Coccia R, Barone E, Head E, Butterfield DA, Di Domenico F (2014) Neuropathological role of PI3K/Akt/mTOR axis in Down syndrome brain. Biochim Biophys Acta 1842 (7):1144-1153. doi:10.1016/j.bbadis.2014.04.007

10. Ali T, Kim T, Rehman SU, Khan MS, Amin FU, Khan M, Ikram M, Kim MO (2018) Natural Dietary Supplementation of Anthocyanins via PI3K/Akt/Nrf2/HO-1 Pathways Mitigate Oxidative Stress, 
Neurodegeneration, and Memory Impairment in a Mouse Model of Alzheimer's Disease. Mol Neurobiol 55 (7):6076-6093. doi:10.1007/s12035-017-0798-6

11. Llanos-Gonzalez E, Henares-Chavarino AA, Pedrero-Prieto CM, Garcia-Carpintero S, Frontinan-Rubio J, Sancho-Bielsa FJ, Alcain FJ, Peinado JR, Rabanal-Ruiz Y, Duran-Prado M (2020) Interplay Between Mitochondrial Oxidative Disorders and Proteostasis in Alzheimer's Disease. Front Neurosci-Switz 13. doi:ARTN 1444

\subsection{9/fnins.2019.01444}

12. Poprac P, Jomova K, Simunkova M, Kollar V, Rhodes CJ, Valko M (2017) Targeting Free Radicals in Oxidative Stress-Related Human Diseases. Trends Pharmacol Sci 38 (7):592-607. doi:10.1016/j.tips.2017.04.005

13. Chen YY, Wang MC, Wang YN, Hu HH, Liu QQ, Liu HJ, Zhao YY (2020) Redox signaling and Alzheimer's disease: from pathomechanism insights to biomarker discovery and therapy strategy. Biomark Res 8:42. doi:10.1186/s40364-020-00218-z

14. Avery SV (2011) Molecular targets of oxidative stress. Biochem J 434 (2):201-210. doi:10.1042/BJ20101695

15. Grant CM, Quinn KA, Dawes IW (1999) Differential protein S-thiolation of glyceraldehyde-3-phosphate dehydrogenase isoenzymes influences sensitivity to oxidative stress. Mol Cell Biol 19 (4):2650-2656. doi:10.1128/MCB.19.4.2650

16. Huang K, Liang Q, Zhou Y, Jiang LL, Gu WM, Luo MY, Tang YB, Wang Y, Lu W, Huang M, Zhang SZ, Zhuang GL, Dai Q, Shen QC, Zhang J, Lei HM, Zhu L, Ye DY, Chen HZ, Zhou L, Shen Y (2019) A Novel Allosteric Inhibitor of Phosphoglycerate Mutase 1 Suppresses Growth and Metastasis of Non-Small-Cell Lung Cancer. Cell Metab 30 (6):1107-1119 e1108. doi:10.1016/j.cmet.2019.09.014

17. Pei L, Kong Y, Shao C, Yue X, Wang Z, Zhang N (2018) Heme oxygenase-1 induction mediates chemoresistance of breast cancer cells to pharmorubicin by promoting autophagy via PI3K/Akt pathway. J Cell Mol Med 22 (11):5311-5321. doi:10.1111/jcmm.13800

18. Liu F, Wu X, Jiang X, Qian Y, Gao J (2018) Prolonged inhibition of class I PI3K promotes liver cancer stem cell expansion by augmenting SGK3/GSK-3beta/beta-catenin signalling. J Exp Clin Cancer Res 37 (1):122. doi:10.1186/s13046-018-0801-8

19. Liu Y, Cao Y, Zhang W, Bergmeier S, Qian Y, Akbar H, Colvin R, Ding J, Tong L, Wu S, Hines J, Chen X (2012) A small-molecule inhibitor of glucose transporter 1 downregulates glycolysis, induces cell-cycle arrest, and inhibits cancer cell growth in vitro and in vivo. Mol Cancer Ther 11 (8):1672-1682. doi:10.1158/1535-7163.MCT-12-0131 
20. Jo H, Mondal S, Tan D, Nagata E, Takizawa S, Sharma AK, Hou Q, Shanmugasundaram K, Prasad A, Tung JK, Tejeda AO, Man H, Rigby AC, Luo HR (2012) Small molecule-induced cytosolic activation of protein kinase Akt rescues ischemia-elicited neuronal death. Proc Natl Acad Sci U S A 109 (26):1058110586. doi:10.1073/pnas.1202810109

21. Wang X, Wang W, Li L, Perry G, Lee HG, Zhu X (2014) Oxidative stress and mitochondrial dysfunction in Alzheimer's disease. Biochim Biophys Acta 1842 (8):1240-1247. doi:10.1016/j.bbadis.2013.10.015

22. Campos-Pena V, Toral-Rios D, Becerril-Perez F, Sanchez-Torres C, Delgado-Namorado Y, Torres-Ossorio E, Franco-Bocanegra D, Carvajal K (2017) Metabolic Syndrome as a Risk Factor for Alzheimer's Disease: Is Abeta a Crucial Factor in Both Pathologies? Antioxid Redox Signal 26 (10):542-560. doi:10.1089/ars.2016.6768

23. Yang S, Zhou F, Ma M, Yuan Y, Zhao S, Yu P (2020) Neuronostatin Promotion Soluble Abeta1-42 Oligomers: Induced Dysfunctional Brain Glucose Metabolism in Mice. Neurochem Res 45 (10):2474-2486. doi:10.1007/s11064-020-03106-y

24. Allen A, Messier C (2013) Plastic changes in the astrocyte GLUT1 glucose transporter and beta-tubulin microtubule protein following voluntary exercise in mice. Behav Brain Res 240:95-102. doi:10.1016/j.bbr.2012.11.025

25. Choeiri C, Staines W, Miki T, Seino S, Messier C (2005) Glucose transporter plasticity during memory processing. Neuroscience 130 (3):591-600. doi:10.1016/j.neuroscience.2004.09.011

26. Kumari U, Heese K (2010) Cardiovascular dementia - a different perspective. Open Biochem J 4:29-52. doi:10.2174/1874091X01004010029

27. <Amyloid b-Peptide Impairs Glucose Transport in Hippocampal and_Cortical Neurons_Involvement of Membrane Lipid Peroxidation.pdf>.

28. Kumar M, Bansal N (2021) Implications of Phosphoinositide 3-Kinase-Akt (PI3K-Akt) Pathway in the Pathogenesis of Alzheimer's Disease. Mol Neurobiol. doi:10.1007/s12035-021-02611-7

29. Zhao Y, Hu X, Liu Y, Dong S, Wen Z, He W, Zhang S, Huang Q, Shi M (2017) ROS signaling under metabolic stress: cross-talk between AMPK and AKT pathway. Mol Cancer 16 (1):79. doi:10.1186/s12943-017-0648-1

30. De Strooper B, Vassar R, Golde T (2010) The secretases: enzymes with therapeutic potential in Alzheimer disease. Nat Rev Neurol 6 (2):99-107. doi:10.1038/nrneurol.2009.218

31. Gwon AR, Park JS, Arumugam TV, Kwon YK, Chan SL, Kim SH, Baik SH, Yang S, Yun YK, Choi Y, Kim S, Tang SC, Hyun DH, Cheng AW, Dann CE, Bernier M, Lee J, Markesbery WR, Mattson MP, Jo DG (2012) Oxidative lipid modification of nicastrin enhances amyloidogenic ?-secretase activity in Alzheimer's disease. Aging Cell 11 (4):559-568

Page 13/15 
32. <Clearance of amyloid $\beta$-peptide.pdf>.

33. Selkoe DJ (2001) Clearing the brain's amyloid cobwebs. Neuron 32 (2):177-180

34. Tanzi RE, Moir RD, Wagner SL (2004) Clearance of Alzheimer's Abeta peptide: the many roads to perdition. Neuron 43 (5):605-608. doi:10.1016/j.neuron.2004.08.024

35. Walia V, Kaushik D, Mittal V, Kumar K, Verma R, Parashar J, Akter R, Rahman MH, Bhatia S, Al-Harrasi A, Karthika C, Bhattacharya T, Chopra H, Ashraf GM (2021) Delineation of Neuroprotective Effects and Possible Benefits of AntioxidantsTherapy for the Treatment of Alzheimer's Diseases by Targeting Mitochondrial-Derived Reactive Oxygen Species: Bench to Bedside. Molecular Neurobiology

36. Winkler EA, Nishida Y, Sagare AP, Rege SV, Bell RD, Perlmutter D, Sengillo JD, Hillman S, Kong P, Nelson AR, Sullivan JS, Zhao Z, Meiselman HJ, Wendy RB, Soto J, Abel ED, Makshanoff J, Zuniga E, De Vivo DC, Zlokovic BV (2015) GLUT1 reductions exacerbate Alzheimer's disease vasculo-neuronal dysfunction and degeneration. Nat Neurosci 18 (4):521-530. doi:10.1038/nn.3966

\section{Figures}

\section{Figure 1}

Increased $A \beta$ and ROS levels in N2a/APP695swe cells

\section{Figure 2}

Decreased GLUT1 expression, the glucose uptake and ATP contents in N2a/APP695swe cells The N2a/WT and N2a/APP695swe cells were collected separately from three different culture flasks and the proteins were extracted for the Western blot analysis. $(a+b)$ Measurement of intracellular glucose uptake and ATP contents. $(c+d)$ Western blot analysis of GLUT1 and GLUT3 proteins. GLUT1 expression was decreased in the APP group and GLUT3 was not significantly changed. Numbers 1, 2, and 3 represent proteins extracted at three different dates. ${ }^{*} \mathrm{P} \leq 0.05,{ }^{*} \mathrm{P} \leq 0.01$, and ${ }^{* \star *} \mathrm{P} \leq 0.001$.

\section{Figure 3}

GLUT1 enhanced the glucose uptake and ATP contents, reduced A levels in N2a/APP695swe cells

\section{Figure 4}


ROS scavenger N-acetyl-L-Cysteine (NAC) increased GLUT1 expression, the glucose uptake and ATP contents, decreased A $\beta$ levels in N2a/APP695swe cells

\section{Figure 5}

Phosphorylation of PI3K/Akt pathway decreased in N2a/APP695swe cells

\section{Figure 6}

NAC upregulated PI3K/Akt/GLUT1 pathway levels, increased glucose uptake and ATP contents, decreased $A \beta$ levels

\section{Figure 7}

Activated PI3K/Akt/GLUT1 increased glucose uptake and ATP contents, decreased A $\beta$ levels in N2a/APP695swe cells

\section{Figure 8}

Schematic: ROS reduces PI3K/Akt pathway phosphorylation levels leading to cellular GLUT1 deficiency and impaired glucose uptake, NAC improves this state 